Case

Report

\title{
Resection of Pulmonary Metastases 12 Years after Initial Surgery for a Benign Pheochromocytoma
}

\author{
Michiko Ueda, MD, ${ }^{1}$ Fumihiro Shoji, MD, PhD, ${ }^{1}$ Yuka Kozuma, MD, PhD, ${ }^{1}$ \\ Gouji Toyokawa, MD, PhD, ${ }^{1}$ Koji Yamazaki, MD, PhD, ${ }^{1}$ Seiya Momosaki, MD, PhD, ${ }^{2}$ \\ and Sadanori Takeo, $\mathrm{MD}, \mathrm{PhD}^{1}$
}

\begin{abstract}
We describe a rare case of newly discovered pulmonary metastases and surgical confirmation 12 years after initial surgery for a pheochromocytoma. A 61-year-old asymptomatic man was referred because of an abnormal shadow in the right lung field upon chest radiography. Computed tomography (CT) showed two well-demarcated tumors in the basal segment of the right lung. Twelve years previously, he underwent right adrenalectomy and was pathologically diagnosed as having a benign pheochromocytoma. Thereafter, he received a medical check-up annually. To confirm the diagnosis of two pulmonary tumors, video-assisted thoracic surgery was done and wedge resection of the right lower lobe completed. Pathology studies revealed these tumors as pulmonary metastases from the pheochromocytoma, which indicated that the true diagnosis was a malignant pheochromocytoma. Patients with a benign pheochromocytoma should continue to undergo careful monitoring for a long time after the initial surgical procedure. Thoracic surgeons should be aware of the possibility of pulmonary metastases even if $>10$ years have passed since initial resection of a benign pheochromocytoma.
\end{abstract}

Keywords: pheochromocytoma, long-term follow-up, pulmonary metastasis, surgery

\section{Introduction}

A pheochromocytoma is a rare, catecholamine-producing neuroendocrine tumor. The estimated prevalence of a pheochromocytoma ranges from $0.005 \%$ to $0.1 \%$ of

${ }^{1}$ Department of Thoracic Surgery, Clinical Research Institute, National Hospital Organization Kyushu Medical Center, Fukuoka, Fukuoka, Japan

${ }^{2}$ Department of Pathology, Clinical Research Institute, National Hospital Organization Kyushu Medical Center, Fukuoka, Fukuoka, Japan

Received: June 1, 2020; Accepted: June 25, 2020

Corresponding author: Fumihiro Shoji, MD, PhD. Department of Thoracic Surgery, Clinical Research Institute, National Hospital Organization Kyushu Medical Center, 1-8-1 Jigyohama, Chuo-ku, Fukuoka, Fukuoka 810-8563, Japan

E-mail: fshoji@surg2.med.kyushu-u.c.jp

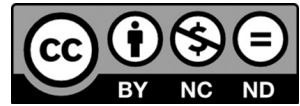

This work is licensed under a Creative Commons Attribution-NonCommercialNoDerivatives International License.

(C)2020 The Editorial Committee of Annals of Thoracic and Cardiovascular Surgery the population. ${ }^{1)}$ This tumor arises mainly from chromaffin cells in the adrenal gland. In general, pheochromocytomas are considered to be benign, but some show malignant characteristics. The prevalence of malignant pheochromocytomas has been reported to be $5-26 \%$ of all pheochromocytomas. ${ }^{2)}$ If this tumor is judged to have not formed distant metastases and has been diagnosed pathologically as a tumor without malignant features, then a method to predict accurately the malignant potential of this tumor is not available. ${ }^{1)}$ Therefore, the diagnosis of a malignant pheochromocytoma can be verified not only at the initial clinical-pathologic confirmation (when the tumor has distant metastases at the initial diagnosis or is diagnosed by pathology to be a malignant pheochromocytoma), but at the time of recognition of tumor recurrence or distant metastases during follow-up after the diagnosis as a benign pheochromocytoma.

We describe a patient with pathology-diagnosed pulmonary metastases 12 years after an initial pathologic diagnosis as a benign pheochromocytoma. 

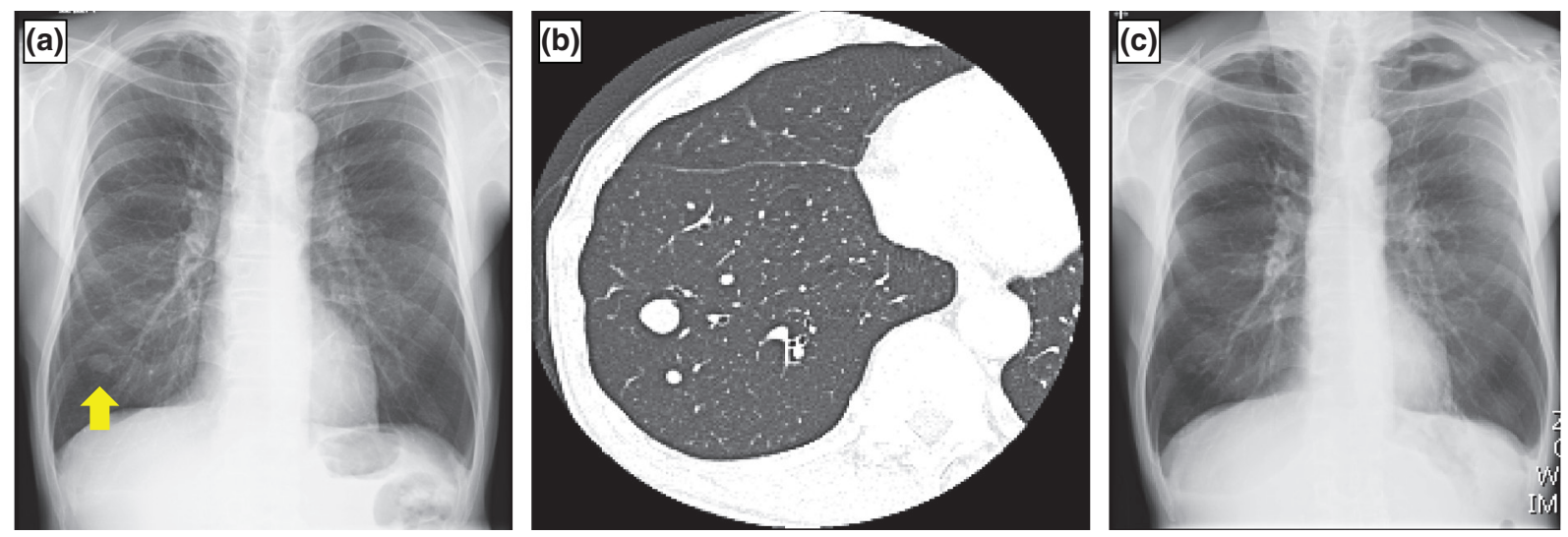

Fig. 1 Time course of chest radiography (a, c). (a) At present, an abnormal shadow in the lower lung field (yellow arrow) was detected, but was not detected 1 year ago (c). CT of the chest at present (b) showed two well-demarcated, round tumors (14 $\mathrm{mm}$ and $5 \mathrm{~mm}$, respectively) in the basal segment of the right lung. CT: computed tomography

\section{Case Report}

A 61-year-old man visited our hospital due to detection of a small abnormal shadow in the right lung field upon chest radiography (Fig. 1a). Contrast-enhanced computed tomography (CT) of the chest showed two well-demarcated, round, heterogeneous-enhanced tumors in the right basal lung (14 $\mathrm{mm}$ and $5 \mathrm{~mm}$, respectively) (Fig. 1b). The patient was asymptomatic. Physical examination (e.g., blood pressure) and laboratory data (including serum and urinary levels of catecholamines) did not show abnormalities.

The patient had been diagnosed as having a benign pheochromocytoma 12 years previously. At that time, a right adrenal tumor measuring $4 \mathrm{~cm}$ according to ultrasound was documented. He was asymptomatic and all laboratory data (including catecholamine levels) were within the normal range. Uptake by ${ }^{123} \mathrm{I}$-meta-iodobenzylguanidine was not found. Based on those examinations, a non-hormonal-functioning pheochromocytoma was suspected. Then, he underwent a right adrenalectomy. Pathology findings showed that the tumor consisted mainly of non-atypical cells with eosinophilic cytoplasm with hemorrhage. Necrosis and mitotic figures were not detected. Finally, the patient was diagnosed as having a non-functioning benign pheochromocytoma. Thereafter, he received an annual medical check-up.

One year ago, the pulmonary lesions described above could not be detected by chest radiography (Fig. 1c). There were no lesions except right-lung nodules according to whole-body CT. Thus, to confirm the diagnosis, video-assisted thoracoscopic surgery was undertaken. The patient was placed in the left lateral position. The thoracoscope was introduced through the seventh intercoastal space on the anterior axillary line. A mini-thoracotomy was done and the right thoracic cavity opened through the fifth intercoastal space. "Elastic-hard" tumors were palpable. Thereafter, wedge resection of the right lower lobe (including nodules with a sufficient surgical margin) was carried out. The macroscopic appearance was welldemarcated yellowish tumors (Fig. 2a). Microscopic examination showed that the well-defined tumors in the resected lung consisted of monotonous polygonal cells arranged in sheets or trabeculae along with vascular stroma. Tumor cells showed moderate nuclear atypia, and mitotic figures were seen occasionally (Fig. 2b). All tumor cells were positive for neuron-specific enolase, cluster of differentiation-56, synaptophysin, S100, and chromogranin A (Figs. 2c-g). These findings were consistent with a pheochromocytoma, which indicated that these pulmonary tumors were distant metastases. Thus, 12 years later, the true diagnosis of this patient was demonstrated to be a malignant pheochromocytoma. The patient was discharged 3 days after the procedure without perioperative complications, and has shown no evidence of tumor recurrence at follow-up.

\section{Discussion}

The "gold standard" therapy for a pheochromocytoma is an adrenalectomy. ${ }^{3)}$ However, benign pheochromocytomas and malignant pheochromocytomas carry the risk of recurrence or metastases. Definitive clinical features or biomarkers to distinguish benign pheochromocytomas from malignant pheochromocytomas are not available. Tumor characteristics (size, location, invasiveness, 


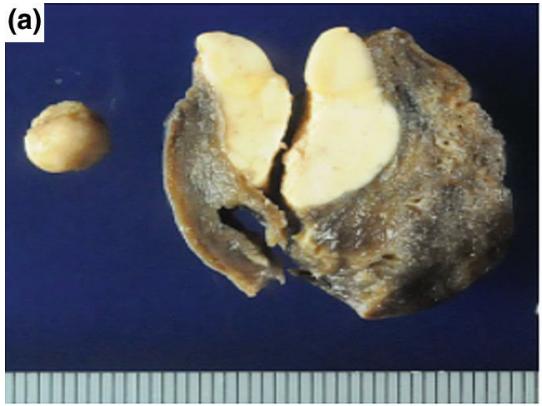

(d)

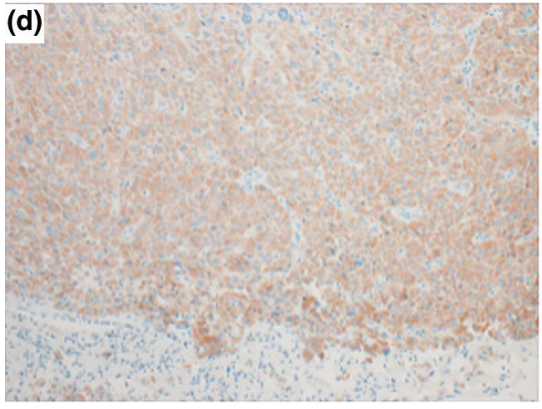

(g)

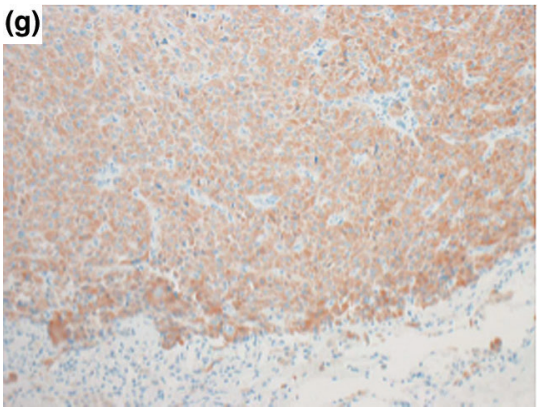

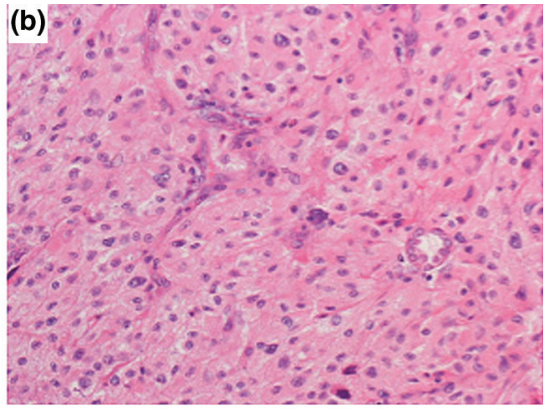
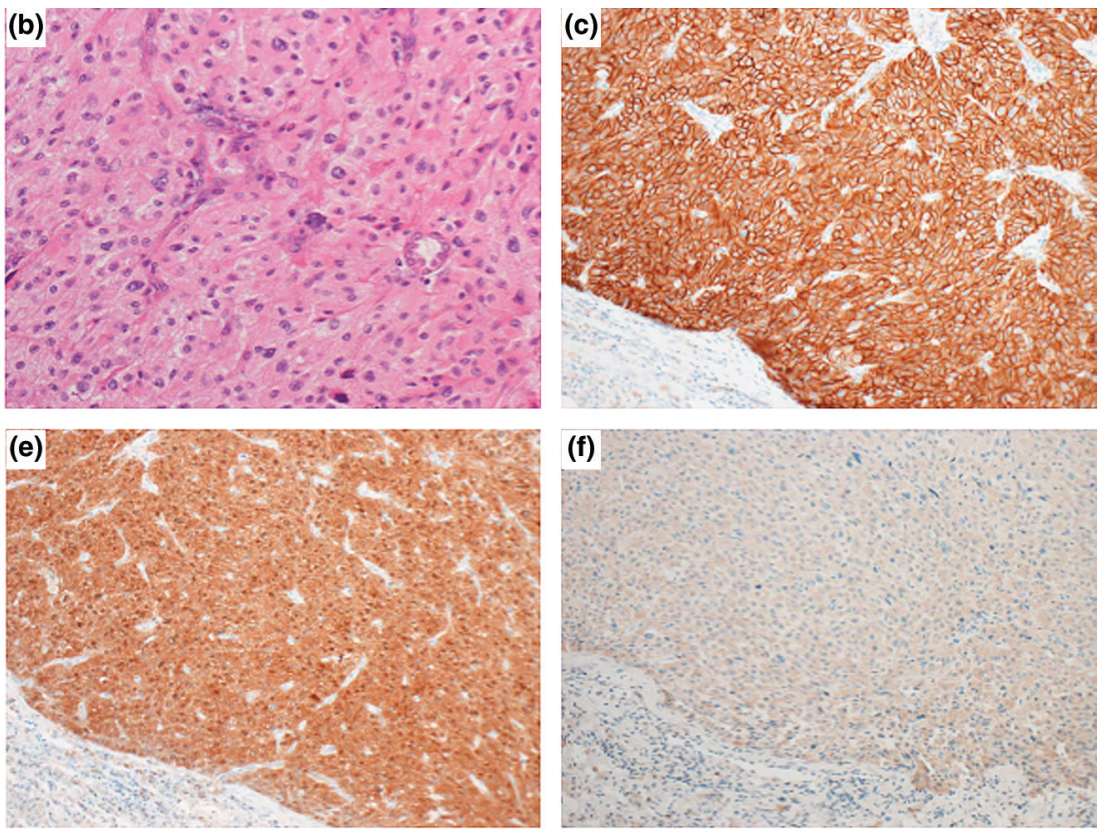

Fig. 2 (a) Macroscopic appearance showed a yellowish surface of the tumor. (b) Pathologic examination revealed well-defined tumors in the resected lung consisting of monotonous polygonal cells arranged in sheets or trabeculae along with vascular stroma. Tumor cells showed moderate nuclear atypia and mitotic figures were seen occasionally (staining with hematoxylin and eosin). Immunohistochemical staining was positive for CD56 (c), chromogranin A (d), neuron-specific enolase (e), S-100 (f), and synaptophysin (g).

necrosis, high cellularity, nuclear pleomorphism, hyperchromasia) might indicate malignant pheochromocytoma, but the widely accepted criterion is distant metastases. ${ }^{4)}$ Therefore, occasionally malignant pheochromocytomas are diagnosed by the discovery of metastases to distant sites where chromaffin cells are absent (e.g., bone, lung, mediastinum, liver, kidney, spleen, lymph nodes) during follow-up. In fact, tumor recurrence or distant metastases occur in $6.5-16.5 \%$ of all pheochromocytoma patients. ${ }^{1)} \mathrm{Kim}$ et al. $\left.{ }^{4}\right)$ reported that 2 of $198(1.0 \%)$ patients with a benign pheochromocytoma had postoperative recurrence. The probability of postoperative recurrence and metastases in a patient with a benign pheochromocytomas may indeed be very low and the diagnosis of a benign pheochromocytoma itself may be misleading. A delay in the diagnosis of tumor recurrence and distant metastases may lead to treatment difficulties, so meticulous follow-up is necessary even if the initial diagnosis is a benign pheochromocytoma. Aggeli et al. ${ }^{5}$ showed that all 37 patients who underwent resection of a benign pheochromocytoma did not have tumor recurrence or distant metastases during at a mean follow-up of 152 months after adrenalectomy. Conversely, Huang et al. ${ }^{6}$ reported a patient in whom distant metastases (bone and lung) were found 10 years after the initial pathologic diagnosis of a benign pheochromocytoma. In addition, clinical-practice guidelines set by the European Society of Endocrinology have suggested follow-up for $\geq 10$ years in all pheochromocytoma patients who have undergone surgery. ${ }^{7)}$ In our case, pulmonary metastases were found 12 years after the diagnosis of a benign pheochromocytoma by resection during annual 
Ueda $M$, et al.

medical check-up. Thus, meticulous and long-term follow-up more than 10 years after the initial surgical procedure, even in patients with a benign pheochromocytoma, is recommended.

\section{Conclusions}

We described a rare case of pulmonary metastases found 12 years after the initial adrenalectomy of a benign pheochromocytoma. Meticulous follow-up for more than 10 years after surgery is required even for patients with a benign pheochromocytoma. Thoracic surgeons should be aware of this possibility if pulmonary nodules are found in patients with a history of a benign pheochromocytoma.

\section{Acknowledgment}

We thank Arshad Makhdum, PhD, from Edanz Group (www.edanzediting.com/ac) for editing a draft of this manuscript.

\section{Ethics Statement}

Written informed consent was obtained from the patient for publishing this case report and accompanying images.

\section{Disclosure Statement}

None declared.

\section{References}

1) Farrugia FA, Martikos G, Tzanetis $P$, et al. Pheochromocytoma, diagnosis and treatment: Review of the literature. Endocr Regul 2017; 51: 168-81.

2) Edström Elder E, Hjelm Skog AL, Höög A, et al. The management of benign and malignant pheochromocytoma and abdominal paraganglioma. Eur J Surg Oncol 2003; 29: 278-83.

3) Press D, Akyuz M, Dural C, et al. Predictors of recurrence in pheochromocytoma. Surgery 2014; 156: 1523-7; discussion 1527-8.

4) Kim KY, Kim JH, Hong AR, et al. Disentangling of malignancy from benign pheochromocytomas/paragangliomas. PLoS ONE 2016; 11: e0168413.

5) Aggeli C, Nixon AM, Parianos C, et al. Surgery for pheochromocytoma: A 20-year experience of a single institution. Hormones (Athens) 2017; 16: 388-95.

6) Huang $\mathrm{KH}$, Chung SD, Chen SC, et al. Clinical and pathological data of 10 malignant pheochromocytomas: long-term follow up in a single institute. Int $\mathbf{J}$ Urol 2007; 14: 181-5.

7) Plouin PF, Amar L, Dekkers OM, et al. European Society of Endocrinology Clinical Practice Guideline for long-term follow-up of patients operated on for a phaeochromocytoma or a paraganglioma. Eur J Endocrinol 2016; 174: G1-G10. 